\title{
Dispharynx nasuta (Nematoda: Acuariidae) em Guira guira e Crotophaga ani (Cuculiformes: Cuculidae) no Estado do Rio Grande do Sul, Brasil
}

\author{
Dispharynx nasuta (Nematoda: Acuariidae) in Guira guira and Crotophaga ani \\ (Cuculiformes: Cuculidae) on Rio Grande do Sul State, Brazil
}

Adriane Bartmann ${ }^{\mathrm{I}}$ Suzana Bencke Amato ${ }^{\mathrm{II}}$

\section{RESUMO}

Os nematóides do gênero Dispharynx (Railliet, Henry \& Sisoff, 1912) parasitam aves de diversas ordens e têm sido estudados, principalmente, em aves de importância econômica devido às lesões que provocam no proventrículo dos hospedeiros. Dispharynx nasuta (Rudolphi, 1819) Stiles \& Hassall, 1920 é registrado em duas espécies de Cuculiformes, anu-branco, Guira guira Gmelin, 1788 e anu-preto, Crotophaga ani Linnaeus, 1758, no Sul do Brasil. Entre 2005 e 2008 foram coletados e necropsiados 120 espécimes de G. guira $e$ 120 espécimes de $\boldsymbol{C}$. ani nos municípios de Cacequi, São Vicente do Sul e Jaguari, Rio Grande do Sul, Brasil. A prevalência de D. nasuta foi de $28,3 \%$ em G. guira e 26,7\%, em C. ani, e a intensidade média de infecção foi de 8,81 ( $D P=18,41$; Amplitude 1 - 82) helmintos/hospedeiro, em G. guira, e de 5,10 (DP=6,17; Amplitude 1 - 30) helmintos/hospedeiro, em C. ani. Já a abundância média de infecção foi de 2,28 $(D P=10,02)$ helminto/hospedeiro, em G. guira, e de 1,32 $(D P=3,82)$ helminto/hospedeiro em C. ani. Análise comparativa entre o sexo, a maturidade sexual e a estação do ano, para as duas espécies de aves, mostrou que em $\boldsymbol{C}$. ani não ocorreram diferenças significativas com relação a qualquer dos parâmetros analisados. Em G. guira as fêmeas estavam mais parasitadas do que os machos $(P=0,039)$; as fêmeas encontravam-se mais parasitadas no inverno $(P=0,008)$; os machos, no verão $(P=0,011)$; os machos jovens estavam mais parasitados do que os adultos $(P=0,015)$. As lesões encontradas no proventrículo das aves parasitadas confirmaram o diagnóstico de hiperplasia adenomatóide do proventrículo com proventriculite granulomatosa. Esta comunicação amplia o número de espécies hospedeiras e a distribuição geográfica do nematóide D. nasuta.

Palavras-chave: helmintos, anu-branco, anu-preto, histopatologia, patologia, distribuição geográfica.

\begin{abstract}
Nematodes of the genus Dispharynx (Railliet, Henry \& Sisoff, 1912) are parasites of several groups of birds which have been primarily studied in birds of economic importance due to the injuries caused in the host proventricle. Dispharynx nasuta (Rudolphi, 1819) Stiles \& Hassall, 1920, was found in two species of Cuculiformes, guira cuckoos, Guira guira Gmelin, 1788 and smooth-billed ani, Crotophaga ani Linnaeus, 1758, in the southern-most State of Brazil. From 2005 to 2008, 120 specimens of G. guira and 120 specimens of C. ani, were collected in Cacequi, São Vicente do Sul and Jaguari, Rio Grande do Sul State, Brazil. The prevalence of $\mathbf{D}$. nasuta in G. guira was $28.3 \%$ and in $C$. ani $26.7 \%$; the average intensity of infection was 8.81 ( $D P=18.41$; Amplitude 1 - 82) helminths/host in G. guira and 5.10 ( $D P=6.17$; Amplitude 1 $30)$ in C. ani; the average abundance of infection was 2.28 $(D P=10.02)$ helminths/host in $\mathbf{G}$. guira, and $1.32(D P=3.82)$ in $C$. ani, respectively. Comparative analysis between the species, sex, sexual maturity and seasonality showed that $\boldsymbol{C}$. ani did not present significant differences with regard to any of the analyzed parameters. Guira guira presented some differences: females had more parasites than males $(P=0.039)$; female birds collected in the winter $(P=0.008)$ and male birds collected in the summer had more parasites $(P=0.011)$; and immature males had more parasites than adult males $(P=0.015)$. The injuries found in the proventricle of the parasitized birds were compatible with adenomatoid hyperplasia and with granulomatosis proventriculitis. This communication expands the number of host species and the geographic distribution of D. nasuta.
\end{abstract}

Key words: helminths, guira cuckoo, smooth-billed ani, histopathology, pathology, geographical distribution.

'Programa de Pós-graduação em Biologia Animal (PPG-BAN), Universidade Federal do Rio Grande do Sul (UFRGS), Porto Alegre, RS, Brasil.

"Departamento de Zoologia e PPGBAN, UFRGS, CP 15014, 91501-970, Porto Alegre, RS, Brasil. E-mail: sbamato@ufrgs.br. Autor para correspondência. 


\section{INTRODUÇÃO}

Os cuculídeos, anu-branco, Guira guira Gmelin, 1788, e anu-preto, Crotophaga ani Linnaeus, 1758 , são originários das regiões tropicais do Velho Mundo e têm distribuição mundial. Eles apresentam comportamento semelhante, formam casais, constroem ninhos coletivos em bandos com aproximadamente 12 indivíduos. A alimentação é, essencialmente, carnívora, sobretudo, gafanhotos capturados ao acompanhar o gado (SICK, 2001). Na Argentina, BELTZER (1995) estudou a dieta de G. guira, que é baseada principalmente em insetos, moluscos, crustáceos, aracnídeos e anfíbios, constituindo categorias secundárias de alimento, totalizando 39 táxons diferentes.

Acuariidae é a única família reconhecida dentro de Acuarioidea, agrupando nematóides que apresentam como característica a complexidade das estruturas cefálicas de fixação, conhecidas como cordões cefálicos (ANDERSON, 1992). O gênero Dispharynx caracteriza-se pela presença de cordões recorrentes, não-anastomosados, com uma pequena papila cervical, machos com espículos desiguais e dissimilares, geralmente, com cinco pares de papilas pós-cloacais (CRAM, 1927). Variações morfológicas sobre o comprimento dos nematóides juvenis e adultos, o comprimento dos cordões cefálicos e dos ramos descendentes dos cordões em relação ao esôfago muscular e a localização da papila cervical são indicadores da idade dos espécimes de D. nasuta (MACKO et al., 1974b).

CHABAUD (1975) e ZHANG et al. (2004) consideraram Dispharynx como subgênero de Synhimantus Railleit, Henry \& Sisoff, 1912, enquanto BARUŠ (1966), BRASIL \& AMATO (1992) e VICENTE et al. (1995) consideraram como um gênero distinto, classificação mantida neste trabalho. VICENTE et al. (1995) citaram quatro espécies do gênero Dispharynx registradas para o Brasil: Dispharynx capitata (Molin, 1860) Cram, 1927, Dispharynx crassissima (Molin, 1860) Cram, 1927, Dispharynx magnilabiata (Molin, 1860) Gendre, 1920 e Dispharynx spiralis (Molin, 1858) Skrjabin, 1916, sendo esta última considerada como um sinônimo júnior de Dispharynx nasuta (Rudolphi, 1819) Stiles \& Hassall, 1920 (GOBLE \& KUNZ 1945).

O gênero Dispharynx foi registrado pela primeira vez em Cuculiformes, com a espécie Dispharynx resticula Canavan, 1929 encontrada em Crotophaga sulcirostris Swainson, 1827, no Zoológico da Filadélfia, EUA(CANAVAN, 1929). Posteriormente, a espécie $D$. resticula foi encontrada parasitando o esôfago e o proventrículo de $\boldsymbol{C}$. ani, em Cuba, sendo considerada uma espécie muito próxima de $\boldsymbol{D}$. nasuta. A distinção proposta para as duas espécies, D. nasuta e D. resticula, baseou-se nas larguras dos cordões cefálicos e dos espículos e em algumas características da espécie. A espécie D. nasuta foi registrada por BARUŠ (1966) somente para hospedeiros Charadriiformes. MACKO et al. (1974a) registraram a presença de D. nasuta parasitando C. ani, em Cuba. Dispharynx nasuta é um nematóide parasito das glândulas de Lieberkühn, do proventrículo das aves, onde mantém a região anterior presa na glândula e a posterior livre no lúmen do órgão. Esse nematóide tem ampla distribuição geográfica. No Brasil, foi registrado em aves com importância econômica (Galliformes) devido às lesões que provoca no órgão, existindo também registros em Columbiformes, Strigiformes, Falconiformes, Charadriiformes e Passeriformes. De acordo com GOBLE \& KUNZ (1945) e ANDERSON (1992), D. nasuta tem como hospedeiros intermediários isópodos terrestres, ortópteros, coleópteros e diplópodos.

Os objetivos deste trabalho são registrar a presença de D. nasuta em duas espécies de Cuculiformes, G. guira e C. ani, no Sul do Brasil, descrever as lesões encontradas no proventrículo das aves hospedeiras e relacionar a intensidade de infecção à estação do ano, ao sexo e à maturidade sexual das aves.

\section{MATERIAL E MÉTODOS}

Cento e vinte anus-brancos (G. guira) e 120 anus-pretos (C. ani) foram coletados, entre 2005 e 2008 , no verão e no inverno, tendo sido capturados com arma de fogo [licença do Exército Brasileiro e do Instituto Brasileiro do Meio Ambiente e Recursos Naturais Renováveis (IBAMA) (No 024/2005 NUFAU, 016/2006 DITEC/RS e 15089-1)] nos municípios de Jaguari

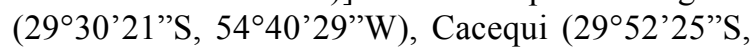
$54^{\circ} 49^{\prime} 31^{\prime \prime}$ ) e São Vicente do Sul (2949'28”, $54^{\circ} 48^{\prime} 07^{\prime}$ 'W), situados na região da Depressão Central do Estado do Rio Grande do Sul (RS), Brasil. Todas as aves foram necropsiadas no Laboratório de Helmintologia, Departamento de Zoologia, Universidade Federal do Rio Grande do Sul (UFRGS), Brasil. Antes da necropsia as aves foram medidas e pesadas. A sexagem foi realizada por identificação das gônadas, e a determinação do estado de maturidade (juvenil e adulto) foi baseada na presença ou ausência da bolsa cloacal (bolsa de Fabricius).

Os procedimentos de coleta e processamento dos helmintos seguiram AMATO \& AMATO (2008). Os nematóides encontrados no interior 
das glândulas do proventrículo e livres no lúmen do órgão foram coletados, fixados em A.F.A. (ácido acético glacial, formalina, álcool etílico) quente e armazenados em etanol $70^{\circ} \mathrm{GL}$. Os espécimes foram clarificados em lactofenol de Amann (HUMASON 1972).

Os espécimes montados in toto, em lactofenol, foram medidos e desenhados. As medidas foram apresentadas em micrometros $(\mu \mathrm{m})$, caso contrário à unidade foi indicada; a amplitude de variação para cada caractere é seguida, entre parênteses, pela média \pm desvio padrão e pelo número de espécimes medidos. Os termos ecológicos como prevalências, intensidade média da infecção, abundância média da infecção e amplitudes das intensidades de infecção seguem BUSH et al. (1997). Espécimes representativos foram depositados em líquido na Coleção Helmintológica do Instituto Oswaldo Cruz (CHIOC), Fundação Oswaldo Cruz, Rio de Janeiro (RJ), e na Coleção de Helmintos do Departamento de Zoologia, Universidade Federal do Rio Grande do Sul (CH-JFA). Espécimes representativos das duas espécies hospedeiras foram depositados na Coleção Ornitológica do Museu de Ciências Naturais, Fundação Zoobotânica do Rio Grande do Sul, Porto Alegre, RS.

As alterações macroscópicas observadas no proventrículo das aves parasitadas provocadas pelo parasito foram descritas. As amostras de tecido com lesões foram fixadas em DUBOSC-Brasil modificado (930 $\mathrm{ml}$ etanol $70^{\circ} \mathrm{GL}, 50 \mathrm{ml}$ formalina, $20 \mathrm{ml}$ ácido acético glacial) e armazenadas em etanol $70^{\circ} \mathrm{GL}$, para estudo histopatológico. Os helmintos e os cortes histológicos foram fotomicrografados em microscópio óptico Zeiss Axiolab. Os desenhos dos helmintos foram realizados com auxílio de tubo de desenho acoplado ao microscópio Nikon E 200. Para análise comparativa entre as espécies, com relação ao sexo, à maturidade sexual e às estações verão e inverno, foi utilizado o teste nãoparamétrico, qui-quadrado $\left(\chi^{2}\right)$ com correção de Yates, quando necessário, com nível de significância de 0,05 , utilizando o programa BioEstat ${ }^{\circledR}$ 5.0 (AYRES et al., 2005).

\section{RESULTADOS E DISCUSSÃO}

Dispharynx nasuta (Rudolphi, 1819) Stiles \& Hassall, 1920

Descrição: Nematóides de cor branca, com dimorfismo sexual, fêmeas maiores que machos. Corpo filiforme, cutícula estriada transversalmente. Lábios pequenos e cônicos. Quatro cordões distintos (Figura 1), recorrentes, não-anastomosados, iniciando na superfície dorsal e ventral da abertura oral, estendendose até a porção posterior do esôfago muscular. Anel nervoso na porção anterior do esôfago muscular. Poro excretor posterior ao anel nervoso. Papilas cervicais bífidas (Figura 7) ao nível do poro excretor. Cápsula bucal estriada transversalmente; esôfago dividido em porção anterior muscular e porção posterior glandular. Machos $(n=24)$ : corpo 7,64 a $11,4 \mathrm{~mm}$ $(9,15 \mathrm{~mm} \pm 1,28 \mathrm{~mm})$ de comprimento, 280 a $360(300 \pm 36,95)$ de largura. Cordões 1,08 a $1,45 \mathrm{~mm}(1,18 \mathrm{~mm} \pm 0,16 \mathrm{~mm})$ de comprimento, 25 a 27,5 $(26,75 \pm 1,21 ; 10)$ de largura. Anel nervoso distando 285 a 357,5 (312,8 $\pm 32,76 \mathrm{~mm})$ da extremidade anterior; poro excretor distando 577,5 a $685(597,9 \pm 61,5 ; 17)$ da extremidade anterior. Papila cervical 710 a $960(751,77 \pm 104,53)$ da extremidade anterior. Distância entre o início do esôfago muscular e a papila cervical 65 a 212,5 (132,73 $\pm 46,79 ; 22)$. Cápsula bucal 207,5 a $275(236,04 \pm 24,86)$ de comprimento, 15 a $17,5(16,25 \pm 1,32 ; 10)$ de largura; distância entre o final da cápsula bucal e o anel nervoso 52,5 a $65(55,42 \pm 6,18)$; esôfago muscular 540 a $830(642,08 \pm 87,08)$ de comprimento, 85 a $117,5(95,31 \pm 13,52)$ de largura; esôfago glandular 2,22 a $3,44 \mathrm{~mm}(2,72 \mathrm{~mm} \pm 0,33 \mathrm{~mm})$ de comprimento, 140 a $200(158,3 \pm 23,67)$ de largura. Extremidade caudal enrolada (Figura 4), com a abertura da cloaca distando 390 a $545(446,98 \pm 55,07)$ da extremidade posterior do corpo. Nove pares de papilas caudais, quatro pré-cloacais e cinco pós-cloacais pedunculares. Distância entre a cloaca e o primeiro par de papilas pós-cloacais 100; distância entre o primeiro par de papilas pós-cloacais e o segundo 57,5; entre o segundo e o terceiro par de papilas 137,5; entre o terceiro e o quarto par de papilas 50; entre o quarto e o quinto par de papilas 92,5; e entre o quinto par de papilas póscloacais e a extremidade posterior da cauda 45. Espículos desiguais e dissimilares, espículo maior e mais fino (Figura 2), 800 a 912,5 (842,19 $\pm 38,41)$ e espículo menor 280 a $322,5(294,27 \pm 19,47)$ de comprimento (Figura 3 ).

Fêmeas $(\mathrm{n}=24)$ : corpo 6,12 a $13,63 \mathrm{~mm}$ $(9,91 \mathrm{~mm} \pm 2,16 \mathrm{~mm})$ de comprimento, 280 a 650 $(442,92 \pm 117,64)$ de largura. Cordões 1,15 a 2,48mm $(1,92 \mathrm{~mm} \pm 0,39 \mathrm{~mm})$ de comprimento, 20 a $25(22,71 \pm 1,29$; 12) de largura. Anel nervoso 235 a 422,5 (344,02 $\pm 50,30$; 23) da extremidade anterior; poro excretor 540 a 1030 $(675,67 \pm 133,97 ; 15)$ da extremidade anterior. Papilas cervicais 630 a $1130(923,75 \pm 155,47)$ da extremidade anterior. Distância entre o esôfago muscular e as papilas cervicais 120 a 232,5 (157,12 $\pm 31 ; 20)$. Cápsula bucal 215 a $325(263,23 \pm 27,28)$ de comprimento, 15 a 22,5 $(19,17 \pm 3,08)$ de largura. Distância entre a cápsula bucal e o anel nervoso 52,5 a 77,5 $(67,11 \pm 7,92 ; 19)$. Esôfago muscular 460 a $970(743,18 \pm 116,43 ; 22)$ de comprimento, 65 a $162,5(108,52 \pm 25,02 ; 22)$ de largura. Esôfago glandular 2,38 a $4,62 \mathrm{~mm}(3,03 \mathrm{~mm} \pm 0,59 \mathrm{~mm} ; 13) \mathrm{de}$ comprimento, 140 a $230(177,37 \pm 24,26 ; 20)$ de largura. 


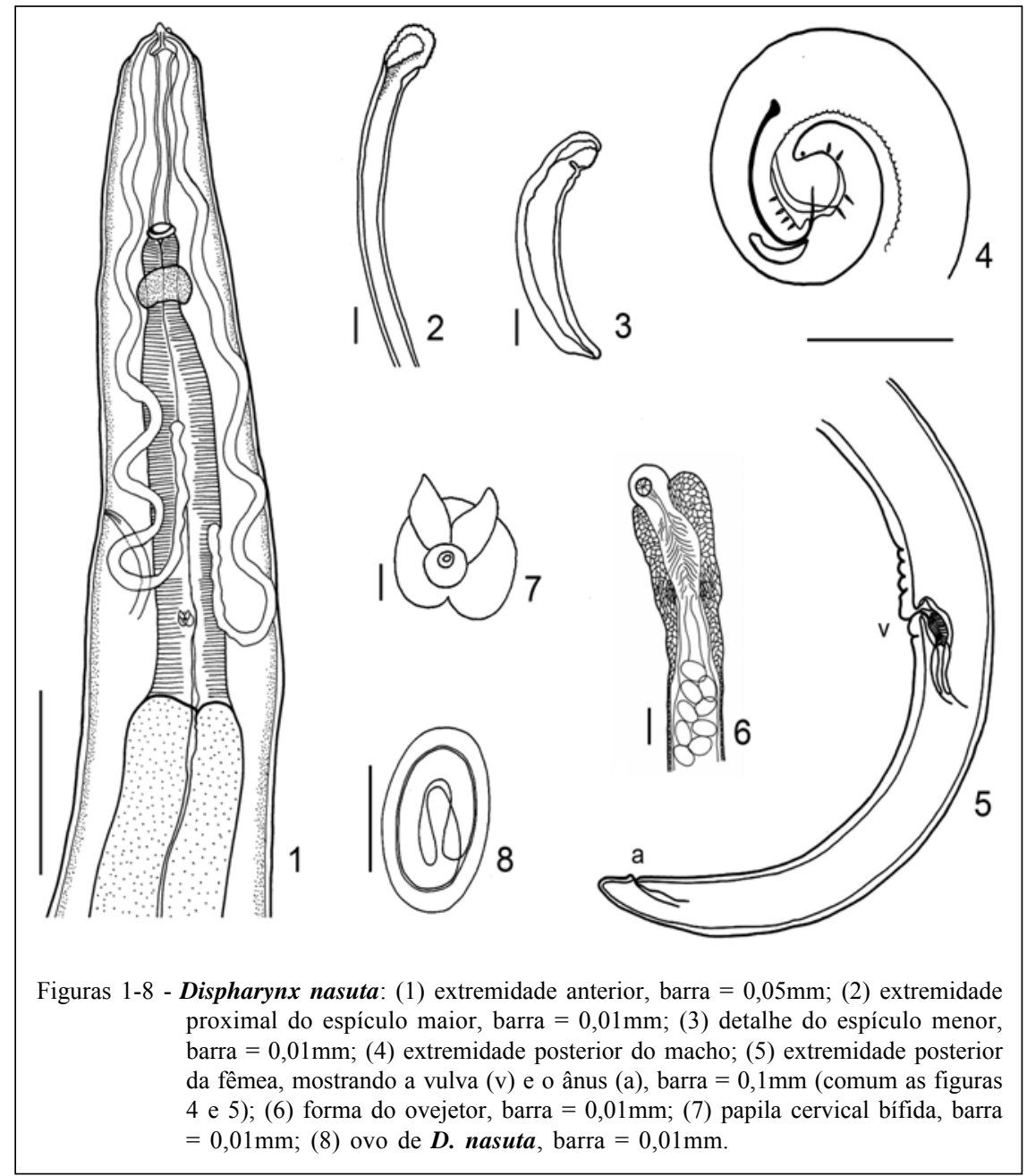

Útero bem desenvolvido, com muitos ovos (Figura 8), 35 a $37,5(35,79 \pm 1,19 ; 19)$ de comprimento, 22,5 de largura, ovejetor (Figura 6) 262,5 de comprimento; vulva (Figura 5) 1,79 a $3,05 \mathrm{~mm}(2,50 \mathrm{~mm} \pm 0,39 \mathrm{~mm})$ da extremidade posterior do corpo. Cauda (Figura 5) 110 a $165(141,46 \pm 14,91)$ de comprimento.

Sinônimos: Spiroptera nasuta Rudolphi, 1819; Dispharagus nasutus (Rudolphi, 1819), Dispharagus nasutus Dujardin, 1844; Dispharagus spiralis Molin, 1858; Filaria nasuta (Rudolphi, 1819) Schneider, 1866; Dispharagus tentaculatus Colucci, 1893; Dispharagus spiralis columbae Bridré, 1910; Acuaria (Dispharynx) nasuta (Rudolphi, 1819) Railliet, Henry \& Sisoff, 1912; Acuaria (Dispharynx) spiralis (Molin, 1858) Railliet, Henry \& Sisoff, 1912; Cheilospirura nasuta (Rudolphi, 1819) Ransom, 1916; Dispharynx spiralis (Molin, 1858) Skrjabin, 1916; Dispharynx stonae Harwood, 1933 (GOBLE \& KUNZ, 1945).
Hospedeiros: anu-branco, Guira guira Gmelin, 1788, e anu-preto, Crotophaga ani Linnaeus, 1758.

Local de infecção: proventrículo.

Prevalências: 28,33\% em G guira, e 26,67\% em C. ani. Intensidades médias de infecção: 8,81 $(\mathrm{DP}=18,41)$ helmintos/hospedeiro, em $\boldsymbol{G}$ guira, e 5,10 $(\mathrm{DP}=6,17)$ helmintos/hospedeiro, em $\boldsymbol{C}$. ani.

Abundâncias médias de infecção: 2,28 $(\mathrm{DP}=10,02)$ helminto/hospedeiro, em G. guira, e 1,32 ( $\mathrm{DP}=3,82)$ helminto/hospedeiro, em C. ani.

Amplitudes das intensidades de infecção: $1-82$ helmintos/hospedeiro, em G. guira, e 1 - 30 helmintos/ hospedeiro, em $\boldsymbol{C}$. ani.

Espécimes representativos depositados: CHIOC no 35605; 35606; 35607 e 35608.

Em geral, o tamanho e as medidas morfométricas dos espécimes coletados em $\boldsymbol{G}$ guira e C. ani foram maiores do que as descritas por CRAM 
(1927), BARUŠ (1966) e ZHANG et al. (2004). Entretanto, essas diferenças podem ser atribuídas ao fato de que medimos, tanto espécimes juvenis, quanto adultos. A característica dos cordões cefálicos, a forma dos espículos, o número e a posição das papilas caudais, a forma do ovejetor, a posição da vulva e a forma e o comprimento da cauda em comparação com a descrição original, confirmam a identificação dessa espécie.

O proventrículo foi o único órgão de infecção de $\boldsymbol{D}$. nasuta nos anus, semelhante aos resultados de BRASIL \& AMATO (1992) e MENEZES et al. (2001), mas a moela também pode ser parasitada por D. nasuta (SPALDING et al., 1996; BITZ et al., 1997). No Brasil, especialmente no Rio Grande do Sul, as criações de aves (galinhas, galinhas d'angola, perus) são realizadas muitas vezes de forma extensiva. Várias espécies de artrópodes são utilizadas como alimento dessas aves e esses artrópodes podem atuar como hospedeiros intermediários de D. nasuta. Os anus são presenças constantes no ambiente peridomiciliar e, com eles, o nematóide D. nasuta. Comparando as prevalências de $\boldsymbol{D}$. nasuta nos anus com as registradas por ROTT et al. (1997), MENEZES et al. (2001), MAGWISHA et al. (2002) e PINTO et al. (2004), a possibilidade de as aves silvestres, anus, estarem envolvidas nas infecções das aves domésticas não pode ser desconsiderada.

Ao comparar as duas espécies de aves quanto à infecção por $\boldsymbol{D}$. nasuta, G. guira apresentou maior prevalência $(28,3 \%)$ do que $\boldsymbol{C}$. ani $(26,7 \%)$, porém essa diferença não foi significativa. A análise comparativa entre as espécies, o sexo, a maturidade sexual e a estação do ano mostrou que Crotophaga ani não apresentou diferenças significativas com relação a nenhum dos parâmetros analisados. Guira guira, entretanto, apresentou algumas diferenças: as fêmeas estavam mais parasitadas do que os machos $(\mathrm{P}=0,039)$; as fêmeas encontravam-se mais parasitadas no inverno $(\mathrm{P}=0,008)$; os machos no verão $(\mathrm{P}=0,011)$; os machos jovens estavam mais parasitados do que os adultos $(\mathrm{P}=0,015)$. As diferenças observadas com relação às fêmeas e à estação do ano devem estar relacionadas à época de reprodução e à alimentação dos filhotes, já que são elas que cuidam dos filhotes e trazem o alimento ao ninho, possibilitando contato com maior número de hospedeiros intermediários. A diferença entre machos juvenis e adultos pode ter-se dado por acaso ou pode estar relacionada ao comportamento ainda desconhecido dessas aves. Porém, em aves juvenis, a maturidade do sistema imunológico é um fator importante diante das infecções parasitárias.
A capacidade patogênica do $\boldsymbol{D}$. nasuta em diversas aves hospedeiras encontra-se estabelecida (GOBLE \& KUTZ, 1945; VASSILEV \& JOOSTE, 1991; BOLETTE, 1998; MENEZES et al., 2001). Os sinais clínicos em galinhas d'angola (Numida meleagris Linnaeus, 1758) infectadas com o nematóide foram detalhados por ROTT et al. (1997), os quais destacaram: desnutrição progressiva, anemia, retardo do crescimento e desenvolvimento em animais juvenis e, em infecções maciças, caquexia e morte da ave em alguns casos.

Os proventrículos parasitados dos anus apresentaram granulomas (Figura 9) de tamanhos variados ( 2 a 9,5mm), geralmente localizados próximos à junção do esôfago. $\mathrm{O}$ tamanho dos nódulos e o grau de inflamação estavam relacionados com o número de nematóides encontrados. Em um espécime de $\boldsymbol{G}$ guira, a intensidade de infecção foi de 82 nematóides, e a lesão causada por eles ocupava dois terços do proventrículo, onde poucos nematóides mantinham a região anterior fixada, enquanto a maioria encontravase livre na luz do órgão. Descrições semelhantes foram realizadas por GOBLE \& KUTZ (1945).

As alterações macroscópicas dos órgãos parasitados incluem significativa dilatação das paredes, excesso de muco no lúmen, nematóides fixados profundamente nas glândulas de Lieberkühn, edema, mucosa espessa, congesta e com projeções papiliformes (Figura 9). Já as alterações histopatológicas observadas no proventrículo foram áreas focais de hiperplasia adenomatóide da mucosa, hiperplasia glandular, leve dilatação cística focal, secreção mucosa, congestão, edema, infiltração de linfócitos e polimorfonucleares na mucosa e submucosa e leve fibrose, confirmando o diagnóstico de hiperplasia adenomatóide do proventrículo com proventriculite granulomatosa (Figura 10).

As prevalências de $\boldsymbol{D}$. nasuta encontradas no presente trabalho estavam acima das encontradas por outros autores. Crotophaga ani e G. guira são registradas pela primeira vez como hospedeiras de $\boldsymbol{D}$. nasuta no Sul do Brasil. Igualmente constitui-se como o primeiro registro a descrição dos achados patológicos. As diferenças encontradas com relação às infecções de $\boldsymbol{D}$. nasuta nas aves analisadas, machos e fêmeas, juvenis e adultos capturados no verão e inverno, devem estar relacionadas ao comportamento de forrageamento das aves, bem como ao desenvolvimento do seu sistema imunológico.

\section{AGRADECIMENTOS}

Ao Prof. J. F. R. Amato, pelas valiosas críticas. À Rosemari T. Oliveira, pelo auxílio técnico na histologia. Ao 

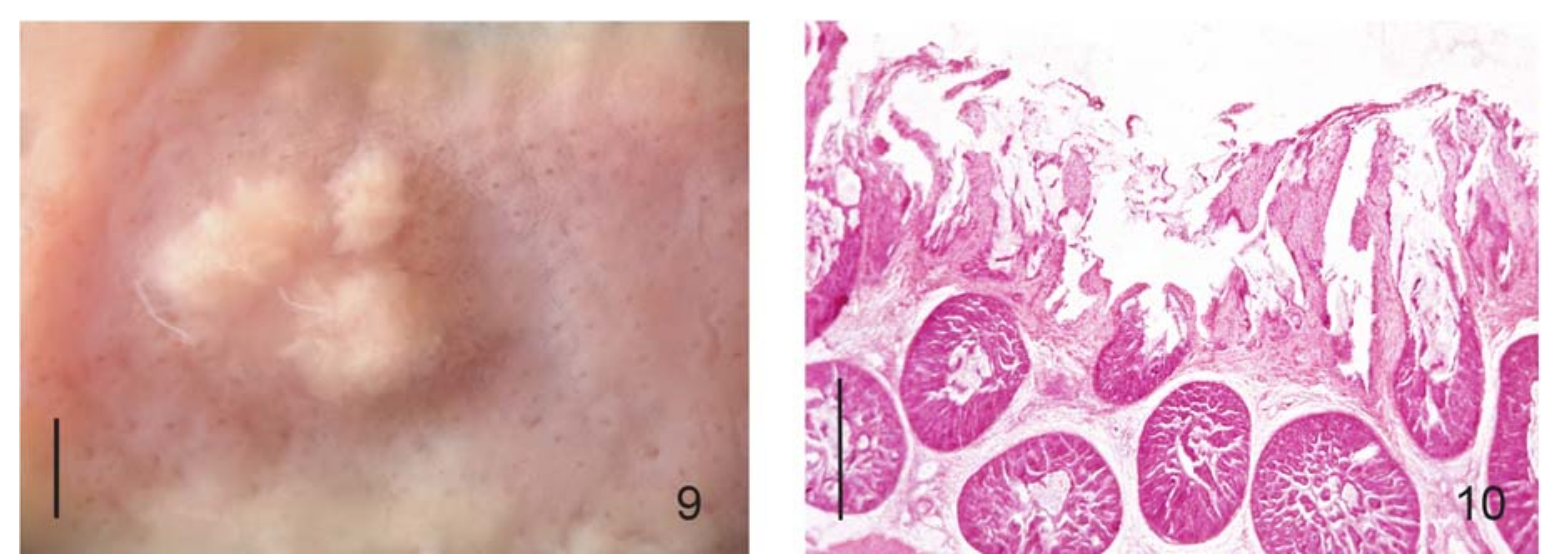

Figuras 9-10 - Proventrículo de G. guira com lesões macro e microscópicas causadas pelo nematóide $\boldsymbol{D}$. nasuta (9) observa-se a lesão causada por Dispharynx nasuta no proventrículo de G. guira, barra $=1 \mathrm{~cm}$; (10) alterações microscópicas observadas no corte histopatológico do proventrículo de G. guira, barra $=500 \mu \mathrm{m}$.

Instituto Brasileiro do Meio Ambiente e dos Recursos Naturais Renováveis (IBAMA), pela licença para coleta dos hospedeiros. Ao Conselho Nacional de Desenvolvimento Científico e Tecnológico $(\mathrm{CNPq})$, pela bolsa de doutorado concedida à primeira autora.

\section{REFERÊNCIAS}

AMATO, J.F.R.; AMATO, S.B. Técnicas gerais para coleta e preparação de helmintos endoparasitos de aves. In: VON MATTER, S. et al. Ornitologia e conservação: ciência aplicada, técnicas de pesquisa e levantamento. Rio de Janeiro: Technical Books, 2008. No prelo.

ANDERSON, R.C. Nematode parasites of vertebrates: their development and transmission. Cambridge: $\mathrm{CAB}$ International, 1992. 578p.

AYRES, M. et al. BioEstat 5.0. Aplicações estatísticas nas áreas das ciências biológicas e médicas. Belém: Sociedade Civil Mamirauá, MCT, Imprensa Oficial do Estado do Pará, 2005. 324p.

BARUŠ, V. Nemátodos parasitos de aves em Cuba. Parte I. Poeyana, Serie A, v.22, p1-37, 1966.

BELTZER, A.H. Biología alimentar del pirincho Guira guira (Aves: Cuculidae) en el Valle aluvial del río Paraná Médio, Argentina. Revista de Ecología Latinoamericana, v.2, p.1318,1995 .

BITZ, M.M.N. et al. Lesiones histopatologicas producidas por Dispharynx nasuta (Nematode: Acuariidae) en Numida meleagris (Aves: Numididae). Veterinária Argentina, v.14, p.401-404, 1997.

BOLETTE, D.P. Dispharynxiasis in a captive Princess Parrot. Journal of Wildlife Diseases, v.34, p.390-391, 1998.

BRASIL, M.C.; AMATO, S.B. Faunistic analysis of the helmints of sparrows (Passer domesticus L., 1758) captured in Campo
Grande, Rio de Janeiro, RJ. Memórias do Instituto Oswaldo Cruz, v.87, p.43-48, 1992.

BUSH, A.O. et al. Parasitology meets ecology on its own terms: Margolis et al. revisited. Journal of Parasitology, v.83, n.4, p.575-583, 1997.

CANAVAN, W.P.N. Nematode parasites of vertebrates in the Philadelphia Zoological Garden and Vicinity. Parasitology, v.21, p.63-102, 1929.

CHABAUD, A.G. Keys to genera of the order Spirurida. Part II. Spiruroidea, Habronematoidea and Acuarioidea. In: ANDERSON R.C., et al. CIH keys to the nematode parasites of vertebrates. Wallingford: CAB International, 1975. N.3, p.29-58.

CRAM, E.B. Bird parasites of the Nematode suborders Strongylata, Ascaridata, and Spirurata. United States National Museum Bulletin, v.140, p.XVIII-456, 1927.

GOBLE, F.C.; KUTZ, H.L. The genus Dispharynx (Nematoda: Acuariidae) in Galliform and Passeriform birds. Journal of Parasitology, v.31, p.323-331, 1945.

Humason, G.L. Animal tissue techniques. San Francisco: W.H. Freeman, 1972. 641p.

MACKO, J.K. et al. The distribution of Dispharynx nasuta and the morphology of $\boldsymbol{D}$. resticula in free-living birds in Cuba. Helminthologia, v.15, p.865-880, 1974a.

MACKO, J.K. et al. On the variability of Dispharynx nasuta (Rudolphi, 1819) in Cuba. Helminthologia, v.15, p.881903, 1974b.

MAGWISHA, H.B. et al. A comparison of the prevalence and burdens of helminth infections in growers and adult free-range chickens. Tropical Animal Health and Production, v.34, p.205-214, 2002. Disponível em: <http:// 
www.springerlink.com/content/vh3wxkvvvadqrvg7/>. Doi: $10.1023 / \mathrm{A}: 1015278524559$.

MENEZES, R.C. et al. Trematodes of free range reared guinea fowls (Numida meleagris Linnaeus, 1758) in the state of Rio de Janeiro, Brazil: morphology and pathology. Avian Pathology, v.30, p.209-214, 2001

PINTO, R.M. et al. First report of five nematode species in Phasianus colchicus Linnaeus (Aves, Galliformes, Phasianidae) in Brazil. Revista Brasileira de Zoologia, v.21, n.4, p.961970, 2004

ROTT, M.I.O. et al. Acuariosis en Numida meleagris (Aves: Numididae). Boletín Chileno de Parasitología, v.52, p.70$72,1997$.

SICK, H. Ornitologia brasileira. Rio de Janeiro: Nova Fronteira, 2001. 912p.
SPALDING, M.G. et al. Helminth and arthropod parasites of experimentally introduced whooping cranes in Florida. Journal of Wildlife Diseases, v.32, p.44-50, 1996.

VASSILEV, G.D.; JOOSTE R. Pathology and taxonomy of Synhimantus (Dispharynx) nasuta infesting bantams and guineafowl in Zimbabwe. Bulletin of Animal Health and Production in Africa, v.39, p.27-30, 1991.

VICENTE, J.J. et al. Nematóides do Brasil. Parte IV. Nematóides de aves. Revista Brasileira de Zoologia, v.12, p.1-273, 1995

ZHANG, L. et al. Two species of Synhimantus (Dispharynx) Railliet, Henry and Sisoff, 1912 (Nematoda: Acuarioidea: Acuariidae), in passerine birds from the Área de Consevacion Guanacaste, Costa Rica. Journal of Parasitology, v.90, p.1133-1138, 2004. 